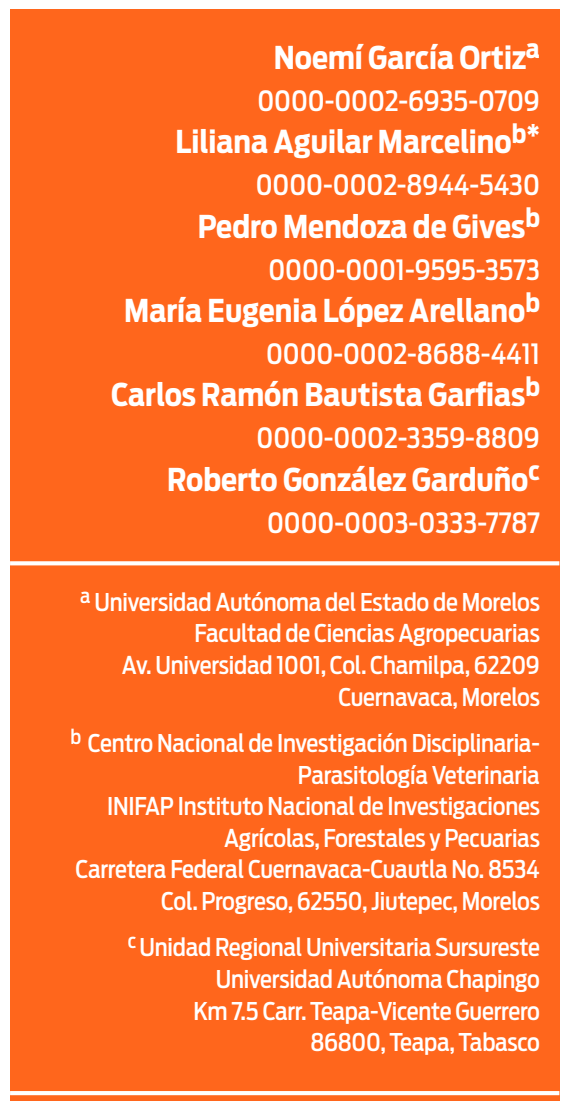

*Autor para correspondencia:

Tel: + 52-77-7319-2860

Correo electrónico:

aguilar.liliana@inifap.gob.mx

Recibido: 2014-06-06

Aceptado: $2015-02-19$

Publicado: $2015-03-19$

Información y declaraciones adicionales en la página 6

(a) Derechos de autor: Noemí García Ortiz et al. 2015

acceso abierto $\boldsymbol{O}$

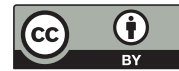

Distribuido bajo una Licencia Creative Commons Atribución 4.0 Internacional (CC-BY 4.0)

\section{Actividad depredadora in vitro de Lasioseius penicilliger (Arachnida: Mesostigmata) contra tres especies de nemátodos: Teladorsagia circumcincta, Meloidogyne sp. y Caenorhabditis elegans}

\begin{abstract}
Resumen
El objetivo de este estudio fue evaluar el comportamiento depredador in vitro del ácaro Lasioseius penicilliger sobre tres especies de nemátodos: Teladorsagia circumcincta $\left(\mathrm{L}_{3}\right.$, nemátodo parásito de ovejas), Meloidogyne sp. $\left(\mathrm{J}_{2}\right.$, nemátodo parásito de plantas), y sobre varios estadios de desarrollo de Caenorhabditis elegans (nemátodo de vida libre). La coincubación de los ácaros y los nemátodos se evaluó en 2\% agar-agua colocados en cajas de Petri de plástico ( $2 \mathrm{~cm} \times 1 \mathrm{~cm}$ diámetro). Se colocaron 1000 nemátodos de cada especie y cinco ácaros en cada caja (10 réplicas) y se incubaron por cinco días a temperatura ambiente $\left(18-25^{\circ} \mathrm{C}\right)$. L. penicilliger mostró comportamiento depredador contra las tres especies de nemátodos evaluadas. Los porcentajes de actividad depredadora registrados fueron 95.1, 80.5 y 79.3, contra Meloidogyne sp., C. elegans y $T$. circumcincta, respectivamente $(P \leq 0.05)$. Estos resultados sugieren que $L$. penicilliger tiene un importante potencial como agente de control biológico contra nemátodos parásitos.
\end{abstract}

Palabras clave: Ácaro depredador; Nemátodos parásitos; Neotropical

\section{Introducción}

Los nemátodos parásitos son responsables de enfermedades graves en plantas y animales. Son una de las principales preocupaciones en la industria ganadera y la agricultura (Fitzpatrick, 2013). Por ejemplo, Teladorsagia circumcincta es uno de los nemátodos parásitos de ovejas económicamente más importantes en las regiones templadas frías (Gossner et al., 2012). Por esta razón, el hombre ha diseñado diferentes productos químicos para el control de nemátodos parásitos; sin embargo, el uso indiscriminado de medicamentos contra nemátodos parásitos y el consiguiente desarrollo y crecimiento de la resistencia a los fármacos en los parásitos, han alentado a los investigadores de todo el mundo a buscar nuevas estrategias de control (Kaplan, 2004; Davies y Spiegel, 2011; Good et al., 2012; Torres-Acosta et al., 2012b).

Por lo tanto, surge la necesidad de utilizar alternativas al control químico, como es el control biológico (Sayre y Walter, 1991; Timper, 2011 ). 
Respecto a la importancia económica de los nemátodos parásitos de rumiantes, se ha hecho hincapié en la necesidad de emplear herramientas moleculares en combinación con métodos convencionales para el diagnóstico de infecciones por nemátodos. Estas herramientas se aplican en las investigaciones de la epidemiología del parásito y de detección de resistencia a los medicamentos (Roeber et al., 2013). Por otra parte, la producción de diferentes cultivos se ve afectada por una variedad de organismos patógenos que perjudican gravemente la producción, desarrollo y vigor de las plantas (Back et al., 2002). Entre estos patógenos se encuentran los fitonemátodos, que causan diversa sintomatología -según el género y la especie-, y son capaces de dañar diferentes partes de la planta. El grado de patogenia depende de la agresividad de la cepa, así como de las adaptaciones anatómicas y fisiológicas de cada planta al parasitismo (Dutta et al., 2011).

En este contexto, Meloidogyne spp., que pertenece al grupo de nemátodos formadores de agallas, se considera una de las plagas más importantes en diversos cultivos, principalmente en los países tropicales y subtropicales, donde estos parásitos están distribuidos in extenso (Luc et al., 2005) y provocan pérdidas económicas anuales severas estimadas en \$125 millones de dólares americanos a nivel mundial (Hodda y Cook, 2009; Safdar y McKenry, 2012; Sikora y Fernandez, 2005).

Como en el caso de los rumiantes, los métodos alternativos de control -como el biológico- urgen para aliviar la enorme carga económica que los nemátodos parásitos causan a la industria de la agricultura (Van der Putten et al., 2006).

Los nemátodos en el suelo tienen varios enemigos naturales, tales como virus, protozoarios (Bjornlund y Ronn, 2008), platelmintos, insectos, tardígrados (Sayre y Walter, 1991), nemátodos "depredadores" de otros nemátodos (Bilgrami, 2008), bacterias, hongos nematófagos (Mendoza de Gives y Torres-Acosta, 2012) y los ácaros (Aguilar-Marcelino et al., 2014). Las especies de los ácaros Mesostigmata del género Lasioseius (Berlese, 1916), que tienen distribución mundial y pertenecen a la familia Ascidae (Ibídem), se consideran depredadores y se encuentran en diversos sustratos, tales como el suelo, la basura y en la asociación con insectos y vertebrados (Walter y Lindquist, 1997).

En particular, la especie L. penicilliger tiene ventajas por sus características como agente potencial de control biológico, tales como su corto ciclo de vida y su reproducción partenogenética, que permiten el aumento de su población a mediano plazo. El L. penicilliger utilizado en este análisis se ha mantenido en el laboratorio durante cinco años alimentado con nemátodos. Esto es importante para seleccionar ácaros con preferencia alimenticia de nemátodos parásitos. Al respecto, existen pocos estudios sobre el uso de los ácaros como agentes de control contra nemátodos parásitos.

El objetivo de esta investigación fue evaluar la actividad depredadora in vitro de $L$. penicilliger sobre T. circumcincta $\left(\mathrm{L}_{3}\right)$, Meloidogyne sp. $\left(\mathrm{J}_{2}\right)$ y C. elegans para probar la hipótesis de que este ácaro se alimenta de manera diferente de los nemátodos en función de su tamaño y de la presencia o no de una cutícula externa. 


\section{Material y métodos}

El origen del material biológico manejado fue el siguiente:

Ácaro

Lasioseius penicilliger (Arachnida: Mesostigmata) se aisló de muestras de suelo en Morelos, México, en 2009 y se identificó de acuerdo con Hughes (1976). Desde entonces, la cepa ácaro se ha mantenido en el laboratorio de Helmintología del CENID-PAVET mediante el cultivo en placas de Petri $(2 \mathrm{~cm}$ de diámetro y $1 \mathrm{~cm}$ de alto) que contienen agar-agua al $2 \%$ a temperatura ambiente $\left(27 \pm 2{ }^{\circ} \mathrm{C}\right)$, en condiciones de oscuridad (Bilgrami, 1994). Una vez por semana, Panagrellus redivivus (Nematoda) se aprovechó como alimento para los ácaros en las placas de cultivo. Los ácaros adultos -tanto machos como hembras- se emplearon al azar.

\section{Nemátodos}

- Teladorsagia circumcincta. Esta especie de nemátodos se aisló de un ciervo parasitado de forma natural en el estado de Guerrero, México, en 2011 (Liébano-Hernández, inédito) y se identificó de acuerdo con sus características morfológicas (Indre et al., 2011 ; Van Wyk et al., 2013). Desde entonces, se ha mantenido como un aislado puro en el CENID-PAVET mediante pases continuos en ovejas jóvenes susceptibles.

- Meloidogyne spp. Este nemátodo se aisló de plantas de tomate infectadas (Lycopersicon esculentum Mill) del municipio de Jojutla, estado de Morelos, México. Esta cepa se cultivó bajo condiciones de invernadero en Jiutepec, Morelos, México, y se mantuvo por pasajes sucesivos en las plantas de tomate en condiciones controladas.

- Caenorhabditis elegans. Se ocupó la cepa N2, variedad Bristol. Este nemátodo se cultivó en placas de Petri que contienen NGM (medio de crecimiento de nemátodos). Como primer paso, una cepa comercial de Escherichia coli silvestre (ER2738, Nueva Inglaterra, Lab Biolabs) se cultivó en NGM durante 2 horas a $37^{\circ} \mathrm{C}$. Una abundante población de nemátodos se logró mediante su transferencia a nuevas placas de Petri que contenían bacterias que crecen en NGM durante tres días (Carvalho et al., 2014).

\section{Diseño experimental}

Para la medición de la capacidad depredadora de los ácaros contra los nemátodos se utilizaron cajas de Petri de plástico ( $2 \mathrm{~cm}$ de diámetro $x 1 \mathrm{~cm}$ de alto). La caja de Petri, con $2 \%$ de agar bacteriológico en agua (WAPD), fue la unidad experimental. Durante el experimento, las cajas se mantuvieron a temperatura ambiente $\left(18-25^{\circ} \mathrm{C}\right)$.

El experimento consistió en colocar cinco L. penicilliger adultos y 1000 larvas de nemátodos en cada WAPD con 10 repeticiones de cada tratamiento. Los tratamientos fueron los siguientes: S1: T. circumcincta $\left(\mathrm{L}_{3}\right)$; S2: Meloidogyne sp. $\left(\mathrm{J}_{2}\right)$; S3: C. elegans. Cada WAPD se incubó durante cinco días y al final de este período los ácaros se separaron de forma manual. Los WAPD se lavaron con agua corriente para recoger las larvas. Después de tres lavados, los nemátodos de cada serie se recuperaron por la técnica del embudo de Baermann después de 12 horas (Thientpont et al., 1986). 
Luego, el conteo de nemátodos se llevó a cabo mediante la colocación de 10 alícuotas de $5 \mu \mathrm{L}$ sobre un portaobjetos de vidrio y se examinó bajo un microscopio óptico (10X). Una vez registrados los datos, la tasa de sobrevivencia y el porcentaje de depredación de $L$. penicilliger en cada especie de nemátodo se calcularon de la siguiente manera:

Tasa de supervivencia $=$ número de larvas recuperadas $/ 1000$

Porcentaje de depredación $=\frac{\text { mnn en el grupo control }- \text { mnn en el grupo tratado }}{m n n \text { en el grupo control }} \times 100$

En donde $m n n=$ media del número de nemátodos.

\section{Análisis estadístico}

Los datos se normalizaron utilizando la transformación de raíz cuadrada y se analizaron mediante un diseño completamente al azar en un arreglo factorial de tratamientos [tres especies de nemátodos $x$ dos niveles de ácaros-(ausencia/presencia)]. Las medias se compararon mediante la prueba de Tukey (SAS, 1998). Un valor de $\mathrm{P} \leq 0.05$ se consideró significativo.

\section{Resultados}

Después de cinco días de coincubación nemátodo-ácaro, el número de larvas recuperadas (media \pm desviación estándar) en el control (C) y grupos tratados (T) fue de $792 \pm 233$ (C) y $164 \pm 262.9$ (T) para T. circumcincta; $415 \pm 11.9$ (C) y $20 \pm$ 34 (T) para Meloidogyne sp., y de $335 \pm 166.7$ (C) y $65 \pm 66$ (T) para C. elegans. Los porcentajes de depredación de L. penicilliger fueron de 79.3\% en T. circumcincta, 95.1\% en Meloidogyne sp. y de 80.5\% en C. elegans (figura 1). Cabe destacar que ninguno de los ácaros murió durante el experimento (datos no mostrados). El cuadro 1 muestra los resultados del análisis de varianza. Como se esperaba, la tasa de supervivencia siempre fue más baja en presencia de los ácaros $(P<0.05)$. En la ausencia de ácaros, T. circumcincta mostró una tasa de supervivencia mayor que la de los otros dos nemátodos, posiblemente porque Meloidogyne sp. y C. elegans estaban completando sus ciclos de vida y no tuvieron alimento durante los cinco días de la prueba, mientras que $T$. circumcincta estaba en su estadio infectivo y no necesitaba comida. En presencia de $L$. penicilliger la tasa de supervivencia de $C$. elegans fue similar a la de Meloidogyne sp. y $T$. circumcincta $(\mathrm{P}<0.05)$, pero la tasa de supervivencia de Meloidogyne sp. fue significativamente menor que la de T. circumcincta $(\mathrm{P}<0.05)$.

\section{Discusión}

Se está considerando a los ácaros como prometedores agentes de control biológico para una serie de plagas agrícolas (Chen et al., 2013). Los resultados de este informe apoyan esta idea con base en que $L$. penicilliger fue capaz de aprovechar- 


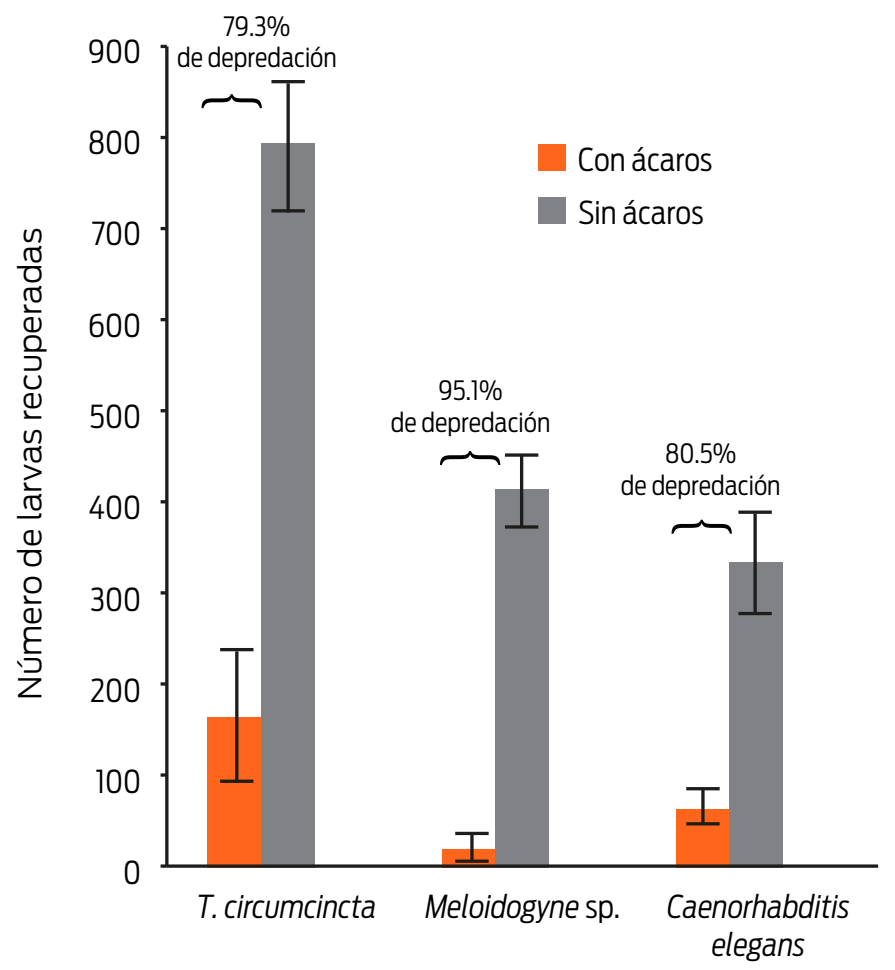

Figura 1. Larvas recuperadas (de 1000) y porcentajes promedio de depredación de L. penicilliger en Teladorsagia circumcinta $\left(\mathrm{L}_{3}\right)$, Meloidogyne sp. $\left(\mathrm{J}_{2}\right)$ y Caenorhabditis elegans (diferentes estadios de desarrollo) después de cinco días de coincubation in vitro. Cada punto representa la media \pm la desviación estándar $(\mathrm{n}=10)$.

Porcentaje de depredación $=[$ (promedio de nemátodos sin ácaros - promedio de nemátodos coincubuados con ácaros) / promedio de nemátodos sin ácaros] x 100. se de los tres diferentes nemátodos evaluados, independientemente de su origen taxonómico.

La tasa de supervivencia de Meloidogyne sp. $\left(\mathrm{J}_{2}\right)$ fue menor que la de $T$. circumcincta $\left(L_{3}\right)$, lo que sugiere un comportamiento depredador selectivo de los ácaros en contra de distintos taxones de nemátodos, lo que podría estar relacionado con las diferencias entre la estructura de la superficie de los nemátodos parásitos de animales y la de nemátodos parásitos de plantas (Gravato-Nobre y Evans, 1998).

Raleigh et al. (1996), por ejemplo, encontraron la presencia de una vaina en las larvas de nemátodos parasitarios de rumiantes, que actúa como una cubierta protectora. Alternativamente, otros miembros del género Lasioseius spp., por ejemplo, L. subterraneus, han demostrado una enorme actividad depredadora contra nemátodos de la raíz (Walter et al., 1993). Sin embargo, hasta ahora hay información muy limitada acerca de los hábitos de alimentación de L. penicilliger como ácaro depredador de nemátodos parásitos de animales.

Recientemente esta especie ha revelado una potencial actividad letal in vitro contra larvas infectantes de $\mathrm{H}$. contortus (Aguilar-Marcelino et al., 2014). Parte de la información sobre la actividad depredadora de otras especies Lasioseius se ha registrado en contra de nemátodos parásitos de las plantas de importancia económica. Por ejemplo, L. scapulatus manifestó 99\% de actividad depredadora in vitro contra Aphelenchus avenae (Imbriani y Mankau, 1983).

Con respecto a la actividad depredadora de L. penicilliger contra nemátodos parásitos de animales, un trabajo reciente in vitro demostró $80 \%$ de depredación de este ácaro contra larvas infectantes de $\mathrm{H}$. contortus (Aguilar-Marcelino et al., 2014). Tales observaciones sugieren que, tal vez, L. penicilliger actúa de manera similar contra otros miembros de la familia Trichostongylidae. Los resultados del presente estudio también indican una alta actividad depredadora de L. penicilliger en las tres especies de nemátodos valoradas.

El hecho de que dos de las especies de nemátodos analizados (T. circumcincta y Meloidogyne sp.) sean patógenas para los rumiantes y las plantas, podría tener una implicación importante en otros estudios en busca de una posible aplicación de agentes de control biológico contra nemátodos parásitos tanto de animales como de plantas.

En este sentido, es importante destacar que el hábitat natural de las larvas infectantes de T. circumcincta y los otros miembros del grupo de nemátodos parásitos de rumiantes es precisamente la materia fecal. Un aumento de la población de ácaros depredadores se ha logrado con el empleo de abono orgánico en estudios en los que se utilizaron los ácaros depredadores para el control de nemátodos de cítricos (El-Banhawy et al., 1997). Por lo tanto, tal vez una de las aplicaciones de 
Cuadro 1. Tasa promedio de supervivencia de Teladorsagia circumcinta $\left(L_{3}\right)$, Meloidogyne sp. $\left(\mathrm{J}_{2}\right)$ y Caenorhabditis elegans (diferentes estadios de desarrollo) después de cinco días de coincubación in vitro con $L$. penicilliger.

\begin{tabular}{|c|c|c|c|}
\hline Especie de nemátodo & Nivel de ácaros & $\mathbf{n}$ & $\begin{array}{c}\text { Tasa } \\
\text { de supervivencial } \\
\text { Promedio } \pm \text { DE }\end{array}$ \\
\hline \multirow{2}{*}{ T. circumcinta } & Ausencia & 10 & $0.79 \pm 0.22^{\mathrm{a}}$ \\
\hline & Presencia & 10 & $0.16 \pm 0.26^{c}$ \\
\hline \multirow{2}{*}{ Meloidogyne sp. } & Ausencia & 10 & $0.42 \pm 0.12^{b}$ \\
\hline & Presencia & 10 & $0.02 \pm 0.03^{d}$ \\
\hline \multirow{2}{*}{ C. elegans } & Ausencia & 10 & $0.34 \pm 0.17^{b}$ \\
\hline & Presencia & 10 & $0.07 \pm 0.07^{\mathrm{cd}}$ \\
\hline
\end{tabular}

los ácaros depredadores, por ejemplo, L. penicilliger, para el control de nemátodos parásitos de rumiantes sería a través de su uso en las heces de campo.

En este momento, sólo se trata de una especulación, porque los resultados se obtuvieron in vitro y se deben tomar con cautela. Por otro lado, el hecho de que $L$. penicilliger haya actuado contra $C$. elegans (un nemátodo de vida libre) podría ser una característica indeseable. Falta mucho por estudiar para establecer con precisión el potencial de los ácaros como agentes de control biológico, ya que L. penicilliger es capaz de alimentarse tanto de nemátodos parásitos de animales como de nemátodos parásitos de plantas.

\section{Conclusiones}

La presente investigación reveló la importante actividad depredadora del ácaro L. penicilliger contra larvas infectantes de T. circumcincta. Hasta la fecha, todavía no está claro cómo un control biológico que use ácaros reduciría la población de larvas parasíticas en el campo.

\section{Financiamiento}

Este estudio fue posible gracias al financiamiento de SAGARPA-CONACYT (Proyecto No. 11990/2005).

\section{Agradecimientos}

Los autores agradecen la participación activa del Dr. Enrique Liébano Hernández en esta investigación antes de su fallecimiento.

\section{Conflictos de interés}

Los autores declaran que no tienen conflictos de interés.

\section{Contribución de los autores}

Noemí García Ortiz y María Eugenia López Arellano: realizaron el experimento, hicieron la revisión crítica y aprobaron el manuscrito para su publicación. 
Liliana Aguilar Marcelino: diseñó el experimento, criticó y aprobó el manuscrito para su publicación.

Pedro Mendoza de Gives: analizó los datos, criticó y aprobó el manuscrito para su publicación.

Carlos Ramón Bautista Garfias: analizó los datos, redactó y aprobó el manuscrito para su publicación.

Roberto González Garduño: revisó el análisis estadístico, redactó y aprobó el manuscrito para su publicación.

\section{Referencias}

1) Aguilar-Marcelino L, Quintero-Martínez MT, Mendoza de Gives PME, LópezArellano ME, Liébano-Hernández E, Torres-Hernández G, González-Camacho JM, Cid del Prado I. 2014. Evaluation of predation of the mite Lasioseius penicilliger (Arachnida: Mesostigmata) on Haemonchus contortus and bacteria-feeding nematodes. Journal of Helminthology, 88:20-23.

2) Back MA, Haydock PPJ, Jenkinson P. 2002. Disease complexes involving plant parasitic nematodes and soil borne pathogens. Plant Pathology, 51:683-697.

3) Bilgrami AL. 1994. Predatory behavior of a nematode feeding mite Tyrophagous putrescentiae (Sarcoptiformes: Acaridae). Fundamental and Applied Nematology, 17:293-297.

4) Bilgrami AL. 2008. Biological control potentials of predatory nematodes. In: A. Ciancio, KG Mukerji (editors) Integrated Management and Biocontrol of Vegetable and Grain Crops Nematodes, Springer.

5) Bjornlund L, Ronn R. 2008. "David and Goliath" of the soil food web-Flagellates that kill nematodes. Soil Biology and Biochemistry, 40:2032-2039.

6) Carvalho S, Phillips CP, Teotónio H. 2014. Hermaphrodite life history and the maintenance of partial selfing in experimental populations of Caenorhabditis elegans. Evolutionary Biology, 14:117. http://www.biomedcentral.com/14712148/14/1 17.

7) Chen $Y L, X u C L, X u X N$, Xie H, Zhang BX, Qin HG, Zhou WQ, Li DS. 2013. Evaluation of predation abilities of Blattisociusdolichus (Acari: Blattisociidae) on a plant-parasitic nematode, Radopholus similis (Tilenchida: Pratylenchidae). Experimental Applied Acarology, 60:289-298.

8) Davies K, Spiegel Y (eds.) 2011. Biological control of plant-parasitic nematodes: building coherence between microbial ecology and molecular mechanisms. Springer.

9) Dutta TK, Stephen JP, Kerry BR, Gaur HS, Curtis RHC. 2011. Comparison of host recognition, invasion, development and reproduction of Meloidogyne graminicola and M. incognita on rice and tomato. Nematology, 13:509-520.

10) El-Banhawy EM, Osman HA, El-Sawaf BM, Afia SI. 1997. Interaction of soil predacious mites and citrus nematodes (parasitic and saprophytic), in citrus orchard under different regime of fertilizers. Effect on the population densities and citrus yield. Anzeiger für Schädlingskunde, Pflanzenschtuz, Umweltschutz, 70:20-23.

11) Fitzpatrick JL. 2013. Global food security: the impact of veterinary parasites and parasitologists. Veterinary Parasitology, 195:233-248.

12) Good B, Hanrahan JP, Theodorus de Waal $D$, Patten $T$, Kinsella A, Oliver LC. 2012. Anthelmintic-resistant nematodes in Irish commercial sheep flocks-the 
state of play. Irish Veterinary Journal, 65(1):21. DOI: 10.1186/2046-0481-6521. http://www.irishvetjournal.org/content/65/1/21.

13) Gossner AG, Venturina VM, Shaw DJ, Pemberton JM, Hopkins J. 2012. Relationship between susceptibility of blackface sheep to Teladorsagia circumcincta infection and an inflammatory mucosa $T$ cell response. Veterinary Research, 43:26 http://www.veterinaryresearch.org/content/43/1/26.

14) Gravato-Nobre MJ, Evans K. 1998. Plant and nematode surfaces: their structure and importance in host-parasite interactions. Nematologica, 44:103-124.

15) Hodda M, Cook DC. 2009. Economic impact unrestricted spread cyst trophic composition. Phytopathology, 99:1987-1393.

16) Hughes AMH. 1976. The mites of stored food and houses. Technical bulletin 9. London, England: Her Majesty's Stationery Office, Ministry of Agriculture, Fisheries and Food.

17) Imbriani IY, Mankau R. 1983. Studies on Lasioseius scapulatus, a mesostigmatid mite predaceous on nematodes. Journal of Nematology, 15:523-528.

18) Indre D, Balint A, Hotea I, Sorescu D, Indre A, Darabus G. 2011. Trichostongyles species and other gastrointestinal nematodes identified in sheep from Timis County. Bulletin UASVM, Veterinary Medicine, 68:171-178. http://journals.usamvcluj.ro/index.php/veterinary/article/viewFile/6897/6159.

19) Kaplan RM. 2004. Drug resistance in nematodes of veterinary importance: a status report. Trends in Parasitology, 20:477-481.

20) Luc M, Sikora RA, Bridge J. 2005. Plant parasitic nematodes in subtropical and tropical agriculture. 2nd ed. London, UK: CAB International. pp. 1-10.

21) Mendoza de Gives P, Torres Acosta JFJ. 2012. Biotechnological use of fungi in the control of ruminant parasitic nematodes. En: Paz Silva A, Sol M (eds.) Fungi: Types Environmental Impact and Role in Disease. Nova Science Publisher, Inc. pp. 389-408.

22) Raleigh MG, Brandon MR, Meeusen E. 1996. Stage-specific expression of surface molecules by the larval stages of Haemonchus contortus. Parasite Immunology, 18:125-132.

23) Roeber F, Jex AR, Gasser RB. 2013. Impact of gastrointestinal parasitic nematodes of sheep, and the role of advanced molecular tools for exploring epidemiology and drug resistance-an Australian perspective. Parasites \& Vectors, 6:153. DOI: 10.1186/1756-3305-6-153 http://www.parasitesandvectors.com/content/6/1/153.

24) Safdar AA, McKenry MV. 2012. Incidence and population density of plant-parasite nematodes infecting vegetable crops and associated yield losses in Punjab Pakistan. Pakistan Journal of Zoology, 44:327-333.

25) SAS Institute. 1998. Statistical software Ver. 6.03. Cary, North Carolina, USA.

26) Sayre RM, Walter DE. 1991. Factors affecting the efficacy on natural enemies of nematodes. Annual Review Phytopathology, 29:149-166.

27) Sikora RA, Fernandez E. 2005. Nematode parasites of vegetables. En: Luc M, Sikora RA, Bridge J (eds.) Plant Parasitic Nematodes in Subtropical and Tropical Agriculture, 2nd ed. Wallingford, UK: CAB International. pp. 319-392.

28) Timper P. 2011. Utilization of biological control for managing plant-parasitic nematode. En: Biological Control of Plant-Parasitic Nematodes. Hokkanan HMT (ed.) Springer Dordrecht Heidelberg London New York. pp. 259-260. 
29) Thienpont D, Rochette F, Vanparijs OFJ. 1986. Diagnosing helminthiasis by coprological examination. 2nd ed. Beerse, Belgium: Janssen Research Foundation. pp. 35-36.

30) Torres-Acosta JFJ, Molento M, Mendoza-de-Gives P. 2012a. Research and implementation of novel approaches for the control of nematode parasites in Latin America and the Caribbean: is there sufficient incentive for a greater extension effort? Veterinary Parasitology, 186:132-42.

31) Torres-Acosta JFJ, Mendoza-de-Gives P, Aguilar-Caballero AJ, Cuéllar-Ordaz JA. 2012b. Anthelmintic resistance in sheep farms: update of the situation in the American continent. Veterinary Parasitology, 189:89-96.

32) Van der Putten WH, Cook R, Costa S, Davies KG, Fargette M, et al. 2006. Nematode interactions in nature: models for sustainable control of nematode pests of crop plants? Advances in Agronomy, 89:227-260.

33) Van Wyk JA, Mayhew E. 2013. Morphological identification of parasitic nematode infective larvae of small ruminants and cattle: a practical lab guide. Onderstepoort Journal of Veterinary Research, 80(1). DOI: 10.4102/ojvr.v80i1.539 http://www.ojvr.org/index.php/ojvr/article/view/539.

34) Walter DE, Kaplan DT, Davis EL. 1993. Colonization of greenhouse nematode cultures by nemathophagous mites and fungi. Supplement to Journal of Nematology, 25(4S): 789-794.

35) Walter DE, Lindquist EE. 1997. Australian species of Lasioseius (Acari: Mesostigmata: Ascidae): the porulosus-group and other species from rainforest canopies. Invertebrate taxonomy, 11(4):525-547. 